\title{
Larval production drives temporal patterns of larval supply and recruitment of a coral reef damselfish
}

\author{
M. G. Meekan*, M. J. Milicich**, P. J. Doherty ${ }^{* *}$ \\ Division of Australian Environmental Studies, Griffith University, Nathan, Brisbane, Queensland 4111, Australia
}

\begin{abstract}
We examined the relative influence of planktonic processes and larval production on the larval supply and recruitment of the damselfish Pomacentrus amboinensis during 2 summers at Lizard Island, northern Great Barrier Reef. Larval production was quantified by monitoring the nest sites of males in windward, lagoon and leeward habitats. Larval supply was estimated using light traps deployed in nearshore waters adjacent to the 3 habitats. Recruitment patterns were back-calculated from the otoliths of newly settled fish collected from small artificial patch reefs constructed in each of the 3 habitats. Temporal patterns of spawning, larval supply and recruitment were correlated when the spawning pattern was lagged by a period equivalent to the incubation time of eggs and the average planktonic life. This coupling occurred despite a change in the pattern of spawning between summers from occasional, large episodes to frequent, smaller pulses spread throughout the summer. Once regular cycles were removed from the data sets by ARIMA (integrated auto-regressive movingaverage) modelling, correlations between patterns declined or became non-significant. This suggests that reproduction has an important influence on the timing of recruitment, but that the magnitude of these events is largely determined within the plankton.
\end{abstract}

\section{INTRODUCTION}

The recruitment patterns of coral reef fishes are thought to be strongly influenced by processes acting during the planktonic life of larvae (Victor 1983a, 1986a, Shulman 1985, Doherty \& Williams 1988, Doherty 1991). Within the plankton, larvae are dispersed by a range of advective processes and undergo very high mortality (Williams et al. 1984, Sale 1990). Both processes may act in a stochastic manner, disrupting any regular patterns in larval production and resulting in a recruitment pattern that is unpredictable and extremely variable (Victor 1983a, 1986a, Williams 1983, Sale 1985, Doherty \& Williams 1988, Doherty 1991).

The small size and extended larval development of

Present addresses:

- GIROQ, Pavillon Vachon, Cité Universitaire, Québec, Canada G1K 7P4

- The Australian Institute of Marine Science, PMB 3, Townsville MC, Queensland 4810, Australia most reef fish do not easily permit direct observation of planktonic dispersal and mortality. However, a recent study that compared the daily reproduction and recruitment of a Caribbean damselfish found that the timing of recruitment was determined by regular (lunar) cycles in the production of larvae (Robertson et al. 1988). Furthermore, episodes of recruitment were only 4 times more variable than the episodes of spawning from which they were produced. These results imply that recruitment patterns of this damselfish are not solely determined by variability in planktonic processes, but are also strongly influenced by patterns of larval production.

Here, we compare the relative influence of reproduction and planktonic processes on temporal patterns of larval supply and recruitment of the damselfish Pomacentrus amboinensis from the Great Barrier Reef (GBR). Like other damselfishes, $P$. amboinensis broods clutches of demersally spawned eggs (Thresher 1984). This behaviour was utilised to provide accurate daily records of reproductive output (Doherty 1980, 1983, Mapstone 1982). 
Light traps (Doherty 1987) were used to quantify the distribution and abundance of Pomacentrus amboinensis within the plankton. These traps collect the older larval stages of reef fishes that are competent or near-competent to settle (Thorrold \& Milicich 1990) and catches have been shown to correlate with recruitment patterns at whole-reef scales (Milicich et al. 1992). Thus, light traps can provide an estimate of larval supply to benthic habitats.

Daily patterns of recruitment were back-calculated from the otoliths of newly settled Pomacentrus amboinensis. This is possible because recruits of this species, in common with many other reef fishes (Pannella 1974, Brothers et al. 1976, Victor 1982), have been shown to deposit daily growth increments in their calcareous otoliths (Pitcher 1987). Because the width of these increments changes abruptly at the time of settlement (Victor 1983b), it is possible to determine, within a day or two, the date of seillement for incividual fish by counting the number of increments between the settlement 'transition mark' and the otolith margin (Meekan in press).

If recruitment patterns are largcly dotcrmined by planktonic processes, we predict that there should be little correlation between the spawning, larval supply and recruitment patterns of Pomacentrus amboinensis at whole-reef scales. However, significant relationships between these variables would suggest either limited dispersal (i.e. a local stock-recruitment relationship) or global effects of reproduction on the recruitment signal.

\section{METHODS}

Reproductive output. In spring, male Pomacentrus amboinensis establish nest sites by removing sand from underneath small pieces of flat coral rubble. Females then lay eggs on the underside of the rubble in dense circular patches, which are guarded by the male until hatching (Mapstone 1982).

In October, active nests of male Pomacentrus amboinensis were identified and replaced with halfround sections of terra-cotta pipe (approximate internal area $450 \mathrm{~cm}^{2}$ ). These artificial nests removed any variability in reproduction associated with nest size, and allowed accurate monitoring of reproductive output. This was done by overturning the pipe and laying a clear plastic sheet over its interior. The area of each clutch within the nest was then traced onto the plastic sheet. As eggs were only laid at dawn (Meekan 1992), the age of each clutch could be determined from the developmental stage of its embryos. The nest was then returned to its original position. Since the minimum time required for development of eggs from spawning to hatching was approximately $84 \mathrm{~h}$ (Mapstone 1982), a daily record of reproductive output was obtained by monitoring all nests at $3 d$ intervals. The areas of individual clutches were calculated from the tracings on the plastic sheets using a computerised digitising pad.

Doherty $(1980,1983)$ and Mapstone (1982) found that similar numbers of clutches were laid in the mapped and unmapped nests of damselfishes. Consequently, it was assumed that mapping did not interfere with reproduction. Repeated tracings of the same clutches showed that this technique gave precise ( $\pm 6.3 \%, 95 \%$ confidence interval) estimates of clutch size (Meekan 1992).

The density of eggs within a nest was estimated by photographing several nests in situ. Nests containing clutches at a variety of developmental stages were photographed together with an object of known area. The resulting slides were then displayed on a screen and counts of egys were made within 3 circular quadrats (to reduce edge effects) of $1 \mathrm{~cm}^{2}$ real area. As densities of eggs did not vary during development (Meekan 1992) estimates were pooled to produce an avcrage density of $182 \pm 36$ eggs $\mathrm{cm}^{-2}$ (95\% confidence interval). This figure was then used to convert the areal measurements to the numbers of eggs produced from individual nests.

Using this sampling technique, the reproductive outputs of Pomacentrus amboinensis were monitored during 2 summers (from 3 November 1988 to 22 January 1989 and from 8 October 1989 to 7 January 1990) at Lizard Island on the northern GBR. Artificial nests were provided for males in the windward, lagoon and leeward habitats (Fig. 1). Ten males were sampled in each of 2 sites in these habitats and all nests were monitored every third day. This provided daily records of reproductive success for a total of 60 males in each summer

Larval supply. Light traps were deployed in the nearshore waters around Lizard Island from 7 November 1988 to 29 January 1989 and from 25 October 1989 to 8 January 1990. Pairs of traps were operated in windward, lagoon and leeward habitats corresponding to the scale at which reproduction was measured. During the first summer, sampling in the lagoon habitat began $9 \mathrm{~d}$ later than that in the windward and leeward habitats.

Within each habitat, traps were deployed approximately $300 \mathrm{~m}$ from shore and were separated from each other by $500 \mathrm{~m}$. All traps were suspended near the surface where the greatest densities of pre-settlement fish are found (Doherty unpubl.). Water depth was $20 \mathrm{~m}$ in the windward habitat and $15 \mathrm{~m}$ in both other habitats.

During each night of sampling, traps were operated in 3 periods $(21: 00$ to $22: 00 \mathrm{~h}, 24: 00$ to $01: 00 \mathrm{~h}$ and 


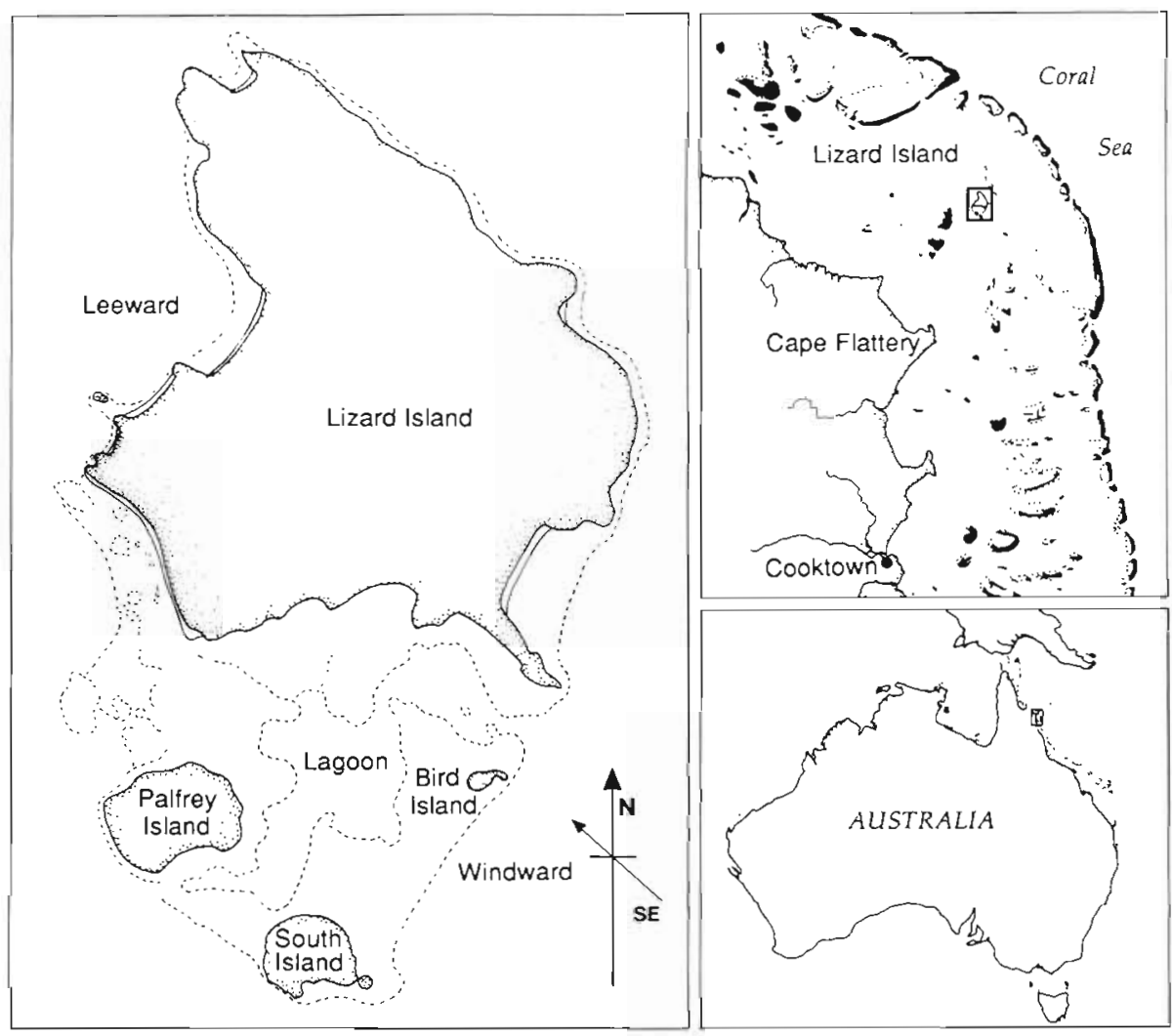

Fig. 1. Lizard Island showing location and reef habitats

During the first summer, recruits were collected at weekly intervals from 5 reefs in each of 3 sites within each habitat. These sites were separated by approximately $250 \mathrm{~m}$. In the second summer, 5 reefs were sampled at weekly intervals at 2 sites, separated by $500 \mathrm{~m}$, within each habitat. Additionally, a further set of 5 reefs were sampled at monthly intervals from 2 sites in each habitat during both summers. All recruits were collected using the anaesthetic Quinaldine ${ }^{\mathrm{r} M \mathrm{M}}$.

The age-analysis of otoliths and the reconstruction of recruitment patterns of Pomacentrus amboinensis have been described by Pitcher (1988) and Meekan (1992, in press). Patterns back-calculated from recruits collected at weekly intervals were significantly correlated with the patterns back-calculated from the monthly col-

03:00 to 04:00 h) to even out any influence of tide or time of night on catch rates. Fish were removed the following morning. At approximately weekly intervals, traps were cleaned and re-deployed on different moorings to eliminate any systematic bias in catches due to variability in the operation of individual traps.

The numbers of traps that operated on any night of sampling varied due to occasional trap failures. Where one of a pair of traps failed to operate, the abundance of pre-settlement fish within that habitat was estimated from the catch of the remaining trap. Both traps within a habitat failed to operate on 6 nights during the 1988-89 summer and on 1 night during the 1989-90 summer. Where traps did not fail on consecutive nights, catches were estimated by interpolating records from the night prior to and following the trap failures.

Recruitment. Newly settled Pomacentrus amboinensis were collected from artificial patch reefs in the windward, lagoon and leeward habitats from 5 November 1988 to 16 February 1989 and from 23 October 1989 to 27 January 1990. Reefs were constructed from Pocillopora coral heads that were collected from a single locality and sun-dried for $24 \mathrm{~h}$. Each reef (approximate dimensions $50 \times 50 \times 35 \mathrm{~cm}$ ) consisted of 3 coral heads placed on the sand at the reef margin. lections (Meekan in press). Consequently, weekly and monthly collections were pooled to increase the sample sizes and thus the resolution of recruitment patterns.

Statistical analyses. Before the coincidence between reproduction, larval supply and back-calculated recruitment could be examined, patterns of spawning had to be lagged by a time period equivalent to incubation and the average planktonic life. For Pomacentrus amboinensis, incubation times ranged between 3.5 and 4.5 d (Mapstone 1982, Meekan 1992), while evidence from otoliths of newly settled individuals suggests that the pelagic stage lasts between 17 and $26 \mathrm{~d}$ with a mean of approximately $19 \mathrm{~d}$ (Brothers et al. 1983, Thresher et al. 1989, Wellington \& Victor 1989). Thus, pre-settlement life in this species for the purposes of these analyses was assumed to be around $23 \mathrm{~d}$.

The coincidence between patterns of spawning, larval supply and recruitment was examined by crosscorrelation analysis (Chatfield 1984). Correlations were initially calculated between raw data sets. ARIMA (integrated auto-regressive moving-average) models were then fitted to the data sets and crosscorrelations calculated between residuals from the models. The seasonally differenced, auto-regressive model $(1,0,0) \times(1,1,0)_{14}$ was fitted to all data sets from the 1988-89 summer and to the spawning data set 
from the 1989-90 summer, while the seasonally differenced, second order auto-regressive model $(2,0,0)$ $\times(1,1,0)_{14}$ was fitted to light trap and back-calculated recruitment data sets from the 1989-90 summer. These ARIMA models removed any regular temporal cycles, so that auto-correlation within the data sets did not influence the cross-correlation analysis. Prior to modelling, all data sets were transformed to square-root values to ensure stationarity of the time series.

Comparisons of data sets at daily intervals may have been influenced by variability introduced by errors in aging (Meekan in press) and changes in the duration of planktonic life of larvae. To account for these potential sources of error, all data sets were pooled to weekly intervals and compared using regression analysis. This reduced the size of the light trap data set to only 20 points and the recruitment data set to only 23 points (pooled between summers). The monthly collections of newly settled Pomacentrus amboinensis were excluded from the recruitment data set before pooling.

Variables were initiaily anaiysed using Model II regression (Bartlett's Linear Functional Relation) because the independent variable (spawning) was subject to both natural variation and measurement error (Laws \& Archie 1981). When bivariate data sets are well correlated, the outcomes from both Model I and Model II regression models converge and in such cases we calculated a predictive (Model I) equation to provide a relative estimate of the explained variance. The regressions were calculated on both raw and transformed data sets. The raw data were transformed by first-differencing to remove any auto-correlation that might have influenced the calculation of coefficients (Thompson \& Page 1989).

\section{RESULTS}

ARIMA modelling identified significant semi-lunar patterns in reproduction during both summers. In the 1988-89 summer, there were 3 major bouts of spawning, the first of which was an extended, possibly bimodal, cycle during November (Fig. 2A). Reproduction in the months of December and January peaked immedi- ately prior to the full moons. In the following summer, the intensity and duration of individual spawning bouts was asymmetric, with strong peaks in reproduction before the full moon and weaker peaks before the new moons (Fig. 3A).

In the 1988-89 summer, the majority of Pomacentrus amboinensis were collected by light traps during December, apparently sourced from the major bout of spawning recorded in November (Fig. 2B). As with the presumptive spawning pattern, the light trap catches were bimodal, with the lowest catches recorded on the new moon. A few larvae were also collected in a single peak after the new moon in January, apparently sourced from the single peak recorded in December. ARIMA modelling identified semi-lunar patterns in light trap catches during the summer, although it is clear that the

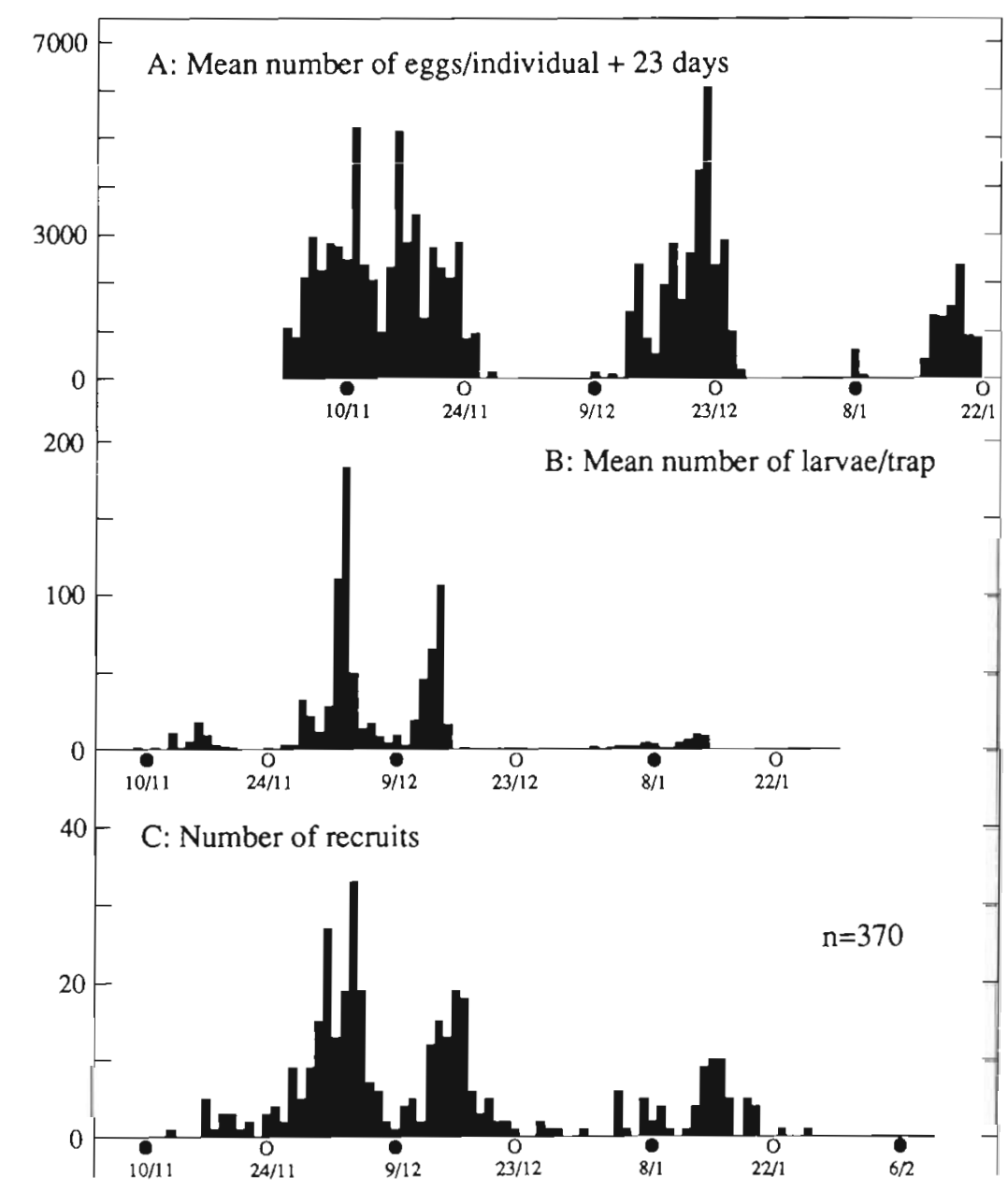

Fig. 2. Pomacentrus amboinensis. Daily records of (A) spawning, (B) light trap catches and $(C)$ back-calculated recruitment of damselfish at Lizard Island during the 1988-89 summer. The spawning pattern has been lagged by $23 \mathrm{~d}$ relative to patterns of light trap catches and recruitment to allow for larval development. Filled circles represent new moons; hollow circles, full moons. Dates given as day/month 


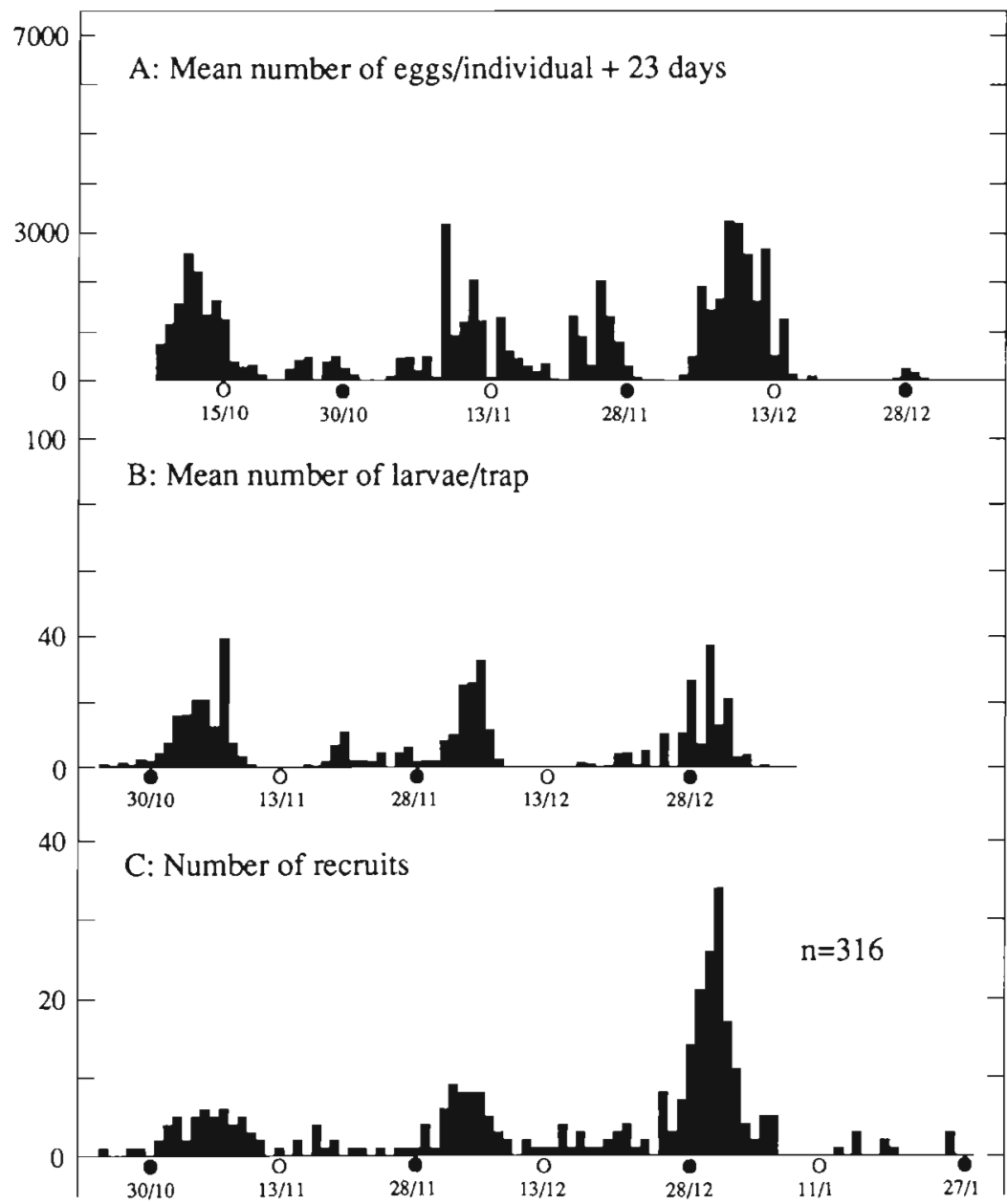

Fig. 3. Pomacentrus amboinensis. Daily records of (A) spawning, (B) light trap catches and $(C)$ back-calculated recruitment of damselfish during the 1989-90 summer. All conventions and symbols follow Fig. 2

relative magnitude of the peaks in light trap catches in these 2 months did not match the sizes of the underlying production cycles (cf. Fig. 2A and 2B).

In the following summer, ARIMA modelling also found semi-lunar patterns in light trap catches, however patterns were dominated by peaks in catches following each of the new moons. Small numbers of fish were caught after the full moons in November and December.

Back-calculated patterns of recruitment were significantly correlated with those of light trap catches in both summers (Table 1). In the 1988-89 summer, there was a large bimodal pulse in recruitment during December with lowest catches around the new moon, although the second peak in this event lagged a few days after the highest catches in light traps (Fig. 2C). Another smaller peak was recorded in the weeks between the new and full moons in January. Again, settlement peaked a few days after light trap catches.
In the following summer, recruitment peaked in the weeks following new moons in October, November and December (Fig. 3C), coinciding with peaks in catches from light traps. The last of these peaks, however, was more intense than either of the preceding peaks, unlike the light trap catches. ARIMA modelling suggested that recruitment during both summers occurred in a semi-lunar pattern.

Cross-correlation analyses of spawning, larval abundance and recruitment patterns are summarised in Table 2. Significant coefficients were calculated between raw data sets in both summers; however, when data sets were modelled and temporal cycles removed, correlations between the variables declined or became nonsignificant. This result implies that the strong correlation found between raw data sets was largely due to the coincident timing of spawning, larval abundance and recruitment patterns.

When data sets were pooled to weekly intervals, strong functional relationships between spawning, larval supply and recruitment were calculated by the Model II regression analyses (Table 3). After first-difference filtering, a significant relationship remained between spawning and recruitment, but not between spawning and light trap catches (Fig. 4). Although it appears that spawning and recruitment are better correlated than spawning and light trap catches $\left(\mathrm{r}^{2}\right.$ for transformed data $=0.51$ and 0.18 respectively) the slopes of both relationships are close to zero and the former comparison seems to be influenced by a few values at each extreme of the line. This suggests that the first-differencing has not completely removed the correlation implicit in comparing 2 cyclic phenomena.

Table 1. Pomacentrus amboinensis, Summary of crosscorrelation analyses comparing daily larval supply with backcalculated recruitment patterns. The second row shows the effect of ARIMA modelling of the data sets prior to analysis. Lag is given in days; ns: non-significant

\begin{tabular}{|lccccc|} 
& \multicolumn{2}{c}{$1988-89$} & \multicolumn{3}{c|}{$1989-90$} \\
& Lag & $\mathrm{r}$ & \multicolumn{2}{c|}{ Lag } & $\mathrm{r}$ \\
\hline Raw & 1 & 0.719 & 0 & 0.576 \\
ARIMA & 2 & 0.358 & & 0 & 0.214 (ns) \\
\hline
\end{tabular}


Table 2. Pomacentrus amboinensis. Summary of crosscorrelation analyses that compared daily spawning with larval supply and back-calculated recruitment patterns. All conventions as in Table 1

\begin{tabular}{|c|c|c|c|c|}
\hline & \multicolumn{2}{|c|}{$1988-89$} & \multicolumn{2}{|c|}{$1989-90$} \\
\hline & Lag & $r$ & Lag & r \\
\hline \multicolumn{5}{|l|}{ Light traps } \\
\hline Raw & -1 & 0.466 & -2 & 0.381 \\
\hline ARIMA & -2 & 0.221 (ns) & 0 & 0.166 (ns) \\
\hline \multicolumn{5}{|c|}{ Recruitment } \\
\hline Raw & 2 & 0.580 & -2 & 0.590 \\
\hline ARIMA & 3 & 0.207 (ns) & -2 & 0.110 (ns) \\
\hline
\end{tabular}

\section{DISCUSSION}

The spawning patterns of Pomacentrus amboinensis clearly had an important influence on patterns of larval supply and recruitment recorded at the scale of Lizard Island. In both summers, there was an obvious temporal concordance between the lagged patterns of reproductive output, light trap catches and backcalculated recruitment patterns. This coupling occurred despite a change in the pattern of spawning from a few broad pulses during 1988-89 to a series of smaller peaks spread over a number of months in the 1989-90 summer. However, correlations between patterns were markedly reduced or became nonsignificant when regular cycles were removed from the data sets by ARIMA modelling. This implies that the reproductive patterns of $P$. amboinensis have a far greater influence on the timing of recruitment events than on their magnitude.

The variations in patterns of recruitment and larval abundance not accounted for by reproduction were presumably caused by planktonic processes, although it is possible that on some occasions the sampling protocol also contributed to the observed variability. As a maximum of only 2 traps were deployed at any

Table 3. Pomacentrus amboinensis. Summary of Model II regression analyses (using Bartlett's Linear Functional Relation) that compared weekly reproductive output with weekly totals of pre-settlement fish collected by light traps and weekly recruitment. Data sets were pooled between summers. Analyses compared both raw and first-difference transformed data sets. $\mathrm{n}=$ number of samples, $a=$ intercept, $b=$ slope of regression, $t$-value $=$ calculated $t$-value

\begin{tabular}{|cccccc|}
\hline & $\mathrm{n}$ & $a$ & $b$ & $t$-value & Probabilities \\
\hline Light traps & & & & & \\
$\quad$ Raw & 20 & -4.107 & 0.0093 & 3.047 & $0.005<\mathrm{p}<0.010$ \\
$\quad$ 1st diff. & 20 & -6.067 & 0.0038 & 1.412 & $0.1<\mathrm{p}<0.200$ \\
Recruitment & & & & & \\
$\quad$ Raw & 23 & 0.638 & 0.0007 & 3.968 & $\mathrm{p}<0.001$ \\
1st diff. & 23 & 0.193 & 0.0006 & 2.818 & $0.01<\mathrm{p}<0.025$ \\
\hline
\end{tabular}

one time in each habitat and these were operated for only $3 \mathrm{~h}$ in each night, some patches of pre-settlement fish in nearshore waters may not have been detected. In addition, newly settled fish were collected from relatively small amounts of habitat $\left(11.25 \mathrm{~m}^{2}\right.$ in the first season and $7.5 \mathrm{~m}^{2}$ in the second). At such scales, recruitment is notoriously patchy (Doherty \& Williams 1988). Both factors may have reduced the correlation between the magnitude of light trap catches and the intensity of recruitment. However, patterns of larval supply and recruitment tended to be more strongly correlated to each other than was either to the observed pattern of spawning.

Asynchrony among patterns was also evident at daily intervals. Prior to the December new moon in the 1988-89 summer, light trap catches peaked a few days before recruitment. Such breakdowns in the concordance of patterns may have occurred for a variety of reasons. Evidence from other light trapping studies on the GBR suggests that the developmental state of presettlement juveniles collected by the traps varies among pulses within a summer (Doherty unpubl.). Consequently, a lag between peaks in light trap catches and recruitment might be expected on some occasions.

Day-to-day variation between larval supply and backcalculated recruitment patterns may have also resulted from errors in the age analysis of otoliths (Meekan in press) since, at best, settlement dates were only determined to within a few days of the event. Additionally. changes in the length of pre-settlement life of Pomacentrus amboinensis may have influenced the concordance of spawning, larval supply and recruitment patterns. During a summer at Lizard Island, the mean time required for incubation of eggs by male $P$. amboinensis declines by $1 \mathrm{~d}$ as higher temperatures promote more rapid development of the embryos, while the duration of pelagic life of a congeneric species ( $P$. nagasakiensis) declines by 2 d (Pitcher 1987, Meekan 1992). This may account for the peak in light trap catches during the new moon in December 1989 occurring a few days prior to the peak in the lagged spawning pattern. Such problems might have been avoided if back-calculated patterns of hatching, rather than recruitment, had been compared with the observed reproduction. However, pre-settlement bands within the central part of otoliths were often difficult to discern, and it was not possible to Ieliably estimate the date of hatching for many recruits. Consequently, we compared patterns of recruitment and reproduction lagged by a fixed period to represent pre-settlement life, although we acknowledge the limitations inherent in this approach. 
Fig. 4. Pomacentrus amboinensis. Plots of weekly reproductive output vs weekly totals of presettlement fish collected by light traps $(A, B)$ and weekly reproductive output vs weekly recruitment $(C, D)$. Plots are shown for raw data sets $(\mathrm{A}, \mathrm{C})$ and the data sets after first-difference transformation (B, D). In all cases the spawning pattern has been lagged by $23 \mathrm{~d}$. All plots show data sets pooled between summers ; squares $=1988-89$ summer, circles $=1989-90$ summer . Solid line shows Model II regression line calculated using Bartlett's Linear Functional Relation. Details of regression analyses are shown in Table 3

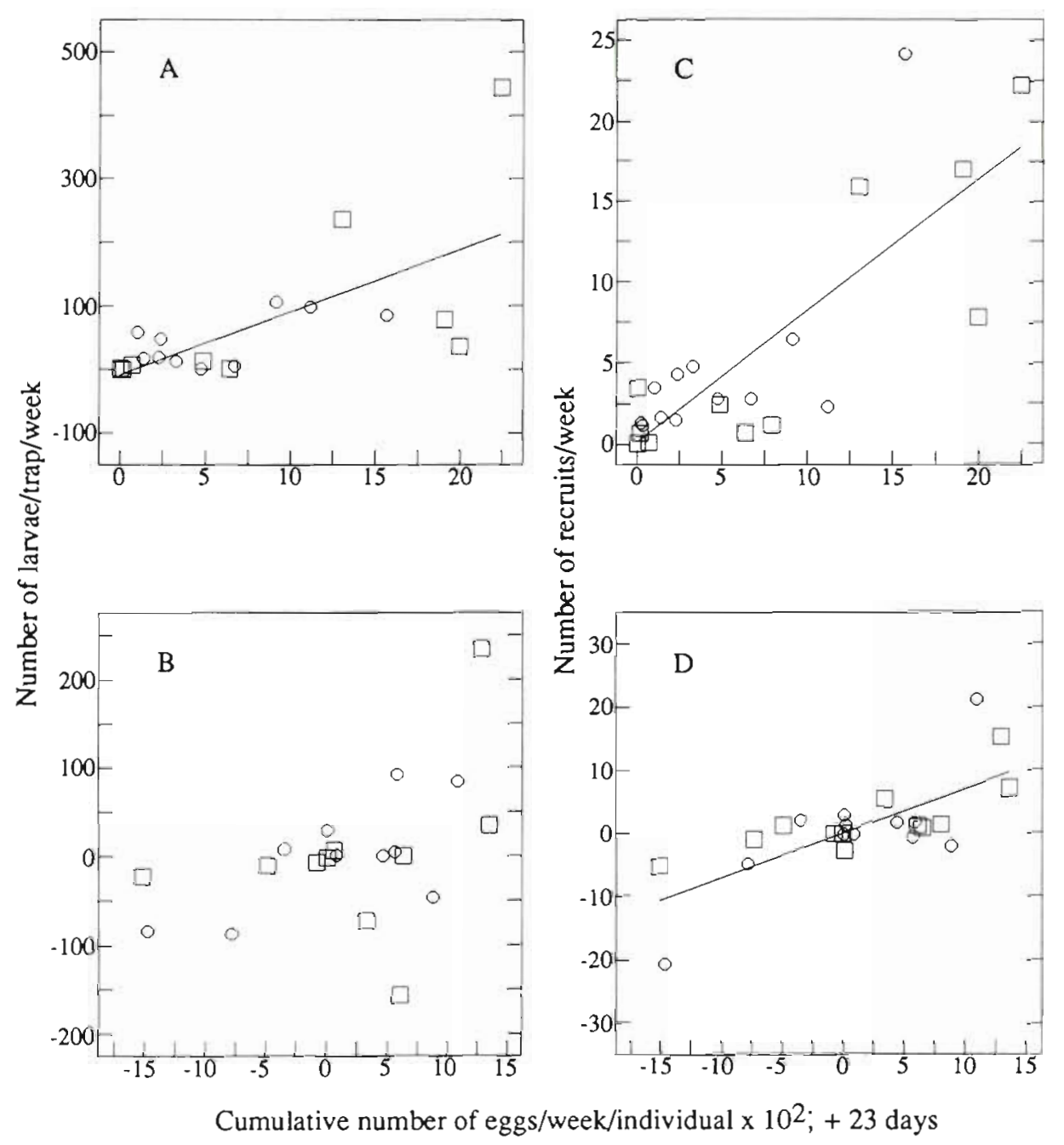

In order to minimise the potential sources of error described above, we pooled spawning, light trap and recruitment signals to weekly intervals and compared the resulting data sets using regression analyses. The weekly data sets were better correlated than the daily time series, which suggests that measurement error may have contributed significantly to asynchrony detected at daily scales. Regression analyses suggested that about half of the variability in recruitment could be explained by spawning patterns; however, this result must be interpreted with caution since the slopes of the regression lines were very close to zero and the upper bounds of the lines were constrained by relatively few points.

Although seasonal and lunar cycles of reproduction and recruitment of reef fishes have been recorded by many previous studies (Johannes 1978, Doherty \& Williams 1988, Robertson 1991), Robertson et al. (1988) performed the only other direct comparison of spawning and recruitment at daily intervals. Similar to this study, Robertson et al. found that lunar patterns in the recruitment of the Caribbean damselfish Stegastes partitus were driven by cycles of the same period in larval production, while the magnitude of peaks in spawning and recruitment of this species was poorly correlated. They concluded that spawning had an important influence on the timing of recruitment, but that the magnitude of recruitment events was largely determined by planktonic processes.

A number of other studies have compared the temporal coupling of spawning and recruitment patterns at less than seasonal scales. Doherty (1991) suggested that the semi-lunar cycles he recorded in the reproduction of the damselfish Pomacentrus wardi on the southern GBR (Doherty 1980, 1983) could account for the semi-lunar recruitment patterns of this species found by Pitcher (1987). Recruitment and spawning were, however, observed on differing reefs, and in differing seasons. In contrast, McFarland et al. (1985) concluded that temporal patterns of spawning could not account for the semi-lunar recruitment of the Caribbean grunt Haemulon flavolineatum. These 
workers suggested that this species spawned in a uniform fashion, and that cycles in recruitment reflected the regular variation in currents favourable to the supply of recruits to benthic habitats. Victor (1983a, 1986a) also argued that the lunar recruitment of the wrasse Thalassoma bifasciatum at Caribbean Panama could not result from the daily pattern of spawning found in this species. However, spawning was not directly measured in either of these studies. McFarland et al. back-calculated spawning patterns from the otoliths of recruits, while Victor relied on the unpublished observations of spawning of $T$. bifasciatum made by other workers. Hunt von Herbing \& Hunte (1991) also found little correlation between the timing of reproduction and recruitment of $T$. bifasciatum in Barbados. While this species spawned in an asymmetric semi-lunar pattern, no significant periodicity was detected in recruitment. Recruitment patterns were, however, back-calculated from individuals collected months after settlement; thus, temporal patterns may have been widely modified by postsettlement mortality. Ochi (1985) recorded acyclical spawning but semi-lunar recruitment in the anemonefish Amphiprion clarkii at a temperate locality in Japan. Doherty (1991) noted that Ochi recorded only the number and not the size of clutches, and that this might have influenced the strength of any correlations between reproduction and recruitment. These difficulties in the interpretation of results are compounded by the problem that the source of recruits arriving at a locality is usually unknown. If the reproductive patterns of populations upcurrent were out of phase or differed from those of the study site, then local patterns of spawning and recruitment could appear to vary independently, even though patterns of recruitment were driven by the reproduction of source populations.

The extent to which larval production determines patterns of larval abundance and recruitment may be a function of the length of the larval life (Hurlbut 1992). A relatively brief planktonic residency may offer little opportunity for planktonic processes to alter production patterns, while a long larval life may result in considerable dispersal and mortality. Unlike the larvae of many reef fishes, which reside in the plankton for months before settlement, the planktonic life of Pomacentrus amboinensis is relatively short and lasts only a few weeks (Brothers et al. 1983, Brothers \& Thresher 1985, Victor 1986b, Thresher et al. 1989, Wellington \& Victor 1989). While this might reduce the influence of planktonic processes, the results of the present study are comparable to those recorded by Robertson et al. (1988) for Stegastes partitus, a species that has a planktonic duration almost twice the length ( $37 \mathrm{~d}$; Robertson et al. 1988) of that of $P$. amboinensis.
At Lizard Island, the correlations between the reproduction and recruitment of Pomacentrus amboinensis may also reflect the geography of the region. Lizard Island lies near the middle of a narrow continental shelf bounded on 3 sides by barriers that are likely to restrict the flow of water and thus the dispersal of larvae (Frith et al. 1986). In other parts of the GBR where the reef matrix is more open and subject to low frequency currents, planktonic larvae may undergo substantially greater levels of mixing and transport, which may uncouple reproduction and recruitment to a greater degree. For example, at One Tree Island on the southern GBR, Doherty (1980) estimated that the annual return of recruitment relative to reproduction of a damselfish varied from $1.10^{5}$ to $1: 10^{6}$ during 3 summers. Unlike Lizard Island, One Tree Reef is not enclosed by a large barrier reef at the edge of the shelf and is a member of a small group of reefs that lie $100 \mathrm{~km}$ to the south of the major reef matrix of the GBR.

While it is tempting to speculate that the close temporal coupling between spawning and recruitment observed at Lizard Island suggests at least a degree of self-recruitment, this argument founders on the lack of information about spawning on surrounding reefs. Given the likely influence of temperature and ecosystem productivity on spawning patterns, it is possible that the correlation between spawning and recruitment may reflect the global nature of the reproduction signal. Distinguishing between these alternatives will require information about the consistency of the production cycle among differing reefs.

The observation that the temporal coupling between reproduction and recruitment persisted between summers, despite a change in the pattern of spawning from a few broad pulses to smaller more numerous peaks, has important implications for the interpretation of recruitment surveys of coral reef fishes. Typically, year-to-year variability in the timing of recruitment pulses within a summer is seen as evidence of the action of processes within the plankton (e.g. Sale 1985, Doherty \& Williams 1988). In the present study, interannual variability in temporal patterns resulted from changes in the timing of spawning. Such results suggest that patterns of spawning of reef fish must be described before the effects of planktonic processes on the timing of recruitment may be interpreted from surveys of recruit abundance.

Acknowledgements. This study was done when M.G.M. and M.J.M. were recipients of Griffith University Postgraduate Scholarships and M.J.M. was a recipient of a Lizard Island Research Fellowship. Other financial and logistical support was provided by the Australian Institute of Marine Science and the Great Barrier Reel Marine Park Authonty. Gilenn Dèath of James Cook University of North Queensland gener- 
ously provided statistical advice. Numerous people assisted with the field work involved in this study; to all, many thanks.

\section{LITERATURE CITED}

Brothers, E. B., Mathews, C. P., Lasker, R. (1976). Daily growth increments in otoliths from larval and adult fishes. Fish. Bull. U.S. 74: 1-8

Brothers, E. B., Thresher, R. E. (1985). Pelagic duration, dispersal, and the distribution of Indo-Pacific coral-reef fishes. In: Reaka, M. L. (ed.) The ecology of coral reefs. NOAA, Rockville, MD, p. 53-69

Brothers, E. B., Williams, D. McB., Sale, P. F. (1983). Length of larval life in twelve families of fishes at 'One Tree Lagoon', Great Barrier Reef, Australia. Mar. Biol. 76: 319-324

Chatfield, C. (1984). The analysis of time series: an introduction. Chapman and Hall, London

Doherty, P. J. (1980). Biological and physical constraints on the populations of two sympatric territorial damselfishes on the southern Great Barrier Reef. Ph.D. thesis, University of Sydney, Sydney

Doherty, P. J. (1983). Diel, lunar and seasonal rhythms in the reproduction of two tropical damselfishes: Pomacentrus flavicauda and P. wardi. Mar. Biol. 75: 215-224

Doherty, P. J. (1987). Light-traps: selective but useful devices for quantifying the distributions and abundances of larval fishes. Bull. mar. Sci. 41: 423-431

Doherty, P. J. (1991). Spatial and temporal patterns in recruitment. In: Sale, P. F. (ed.) The ecology of fishes on coral reefs. Academic Press, San Diego, p. 261-293

Doherty, P. J., Williams, D. McB. (1988). The replenishment of coral reef fish populations. Oceanogr. mar. Biol. A. Rev. 26: $487-551$

Frith, C. A., Leis, J. M., Goldman, B. (1986). Currents in the Lizard Island region of the Great Barrier Reef and their relevance to potential movements of larvae. Coral Reefs 5 : $267-272$

Hunt von Herbing, I., Hunte, W. (1991). Spawning and recruitment of the bluehead wrasse Thalassoma bifasciatum in Barbados, West Indies. Mar. Ecol. Prog. Ser. 72: 49-58

Hurlbut, C. J. (1992). Larval release and supply predict temporal variation in settlement of a colonial ascidian. Mar. Ecol. Prog. Ser. 80: 215-219

Johannes, R. E. (1978). Reproductive strategies of coastal marine fishes in the tropics. Envir. Biol. Fish. 3: 65-84

Laws, E. A., Archie, J. W. (1981). Appropriate use of regression analysis in marine biology. Mar. Biol. 65: 13-16

Mapstone, B. D. (1982). Some aspects of the reproductive ecology of six species of damselfish (Family: Pomacentridae) at One Tree Reef, Great Barrier Reef. Honors thesis, University of Sydney, Sydney

McFarland, W. N., Brothers, E. B., Ogden, J. C., Shulman, M. J., Bermingham, E. L., Kotchian-Prentiss, N. M. (1985). Recruitment patterns in young French grunts, Haemulon flavolineatum (Family Haemulidae), at St. Croix, Virgin Islands. Fish. Bull. U.S. 83: 413-426

Meekan, M. G. (1992). The influence of pre- and post-settlement processes on the population dynamics of coral reef damselfishes. Ph.D. thesis, Griffith University, Brisbane

Meekan, M. G. (in press). Limitations to the back-calculation of recruitment patterns from otoliths. In: Proc. 7 th Int. Coral Reef Symp., Guam

Milicich, M. J., Meekan, M. G., Doherty, P. J. (1992). Larval supply: a good predictor of recruitment in three species of reef fish (Pomacentridae). Mar. Ecol. Prog. Ser. 86: 153-166

Ochi, H. (1985). Temporal patterns of breeding and larval settle- ment in a temperate population of the tropical anemonefish. Amphiprion clarkii. Jap. J. Ichthyol. 32: 248-257

Pannella, G. (1974). Otolith growth patterns: an aid in age determination in temperate and tropical fishes. In: Bagenal, A. B. (ed.) The ageing of fish. Unwin Bros., Gresham Press, Surrey, p. 28-39

Pitcher, C. R. (1987). Validation and application of otolith ageing techniques to some problems in the ecology of coral reef fishes. Ph.D. thesis, Griffith University, Brisbane

Pitcher, C. R. (1988). Validation of a technique for reconstructing daily patterns in the recruitment of coral reef damselfish. Coral Reefs 7: 105-111

Robertson, D. R. (1991). The role of adult biology in the timing of spawning of tropical reef fish. In: Sale, P. F. (ed.) The ecology of fishes on coral reefs. Academic Press, San Diego, p. $356-386$

Robertson, D. R., Green, D. G., Victor, B. C. (1988). Temporal coupling of production and recruitment of larvae of a Caribbean reef fish. Ecology 69: 370-381

Sale, P. F. (1985). Patterns of recruitment of coral reef fishes. In: Gabrié, C., et al. (eds.) Proc. Sth Int. Coral Reef Symp. Antenne Museum - EPHE, Moorea, French Polynesia, p. $391-396$

Sale, P. F. (1990). Recruitment of marine species: is the bandwagon rolling in the right direction? Trends Ecol. Evol. 5: 25-27

Shulman, M. J. (1985). Variability in recruitment of coral reef fishes. J. exp. mar. Biol. Ecol. 89: 205-219

Thompson, K. R., Page, F. H. (1989). Detecting synchrony of recruitment using short autocorrelated time series. Can. J. Fish. Aquat. Sci. 46: 1831-1838

Thorrold, S. R., Milicich, M. J. (1990). Comparison of larval duration and pre- and post-settlement growth in two species of damselfish, Chromis atripectoralis and Pomacentrus coelestis (Pisces: Pomacentridae), from the Great Barrier Reef. Mar. Biol. 105: 375-384

Thresher, R. E. (1984). Reproduction in reef fishes. T. F. H. Publications, Neptune City, NJ

Thresher, R. E., Colin, P. L., Bell, L. J. (1989). Planktonic duration, distribution and population structure of western and central Pacific damselfishes (Pomacentridae). Copeia 1989: $420-434$

Victor, B. C. (1982). Daily growth increments and recruitment in two coral reef wrasses, Thalassoma bifasciatum and Halichoeres bivittatus. Mar. Biol 71: 203-208

Victor, B. C. (1983a). Recruitment and population dynamics of a coral reef fish. Science 219: 419-420

Victor, B. C. (1983b). Settlement and larval metamorphosis produce distinct marks on the otoliths of the slippery dick, Halichoeres bivittatus. In: Reaka, M. L. (ed.) The ecology of deep and shallow coral reefs. NOAA., Rockville, MD, p 47-51

Victor, B. C. (1986a). Larval settlement and juvenile mortality in a recruitment-limited coral reef fish population. Ecol. Monogr. 56: 145-160

Victor, B. C. (1986b). Duration of the planktonic larval stage of one hundred species of Pacific and Atlantic wrasses (family Labridae). Mar. Biol. 90: 317-326

Wellington, G. M., Victor, B. C. (1989). Planktonic larval duration of one hundred species of Pacific and Atlantic damselfishes (Pomacentridae). Mar. Biol. 101: 557-567

Williams, D. McB. (1983). Daily, monthly and yearly variability in recruitment of a guild of coral reef fishes. Mar. Ecol. Prog. Ser. 10: 231-237

Williams, D. McB., Wolanski, E, Andrews, J. C. (1984). Transport mechanisms and the potential movement of planktonic larvae in the central region of the Great Barrier Reef. Coral Reefs 3: 229-236 